\title{
Papers
}

\section{Depression and unwanted first pregnancy: longitudinal cohort study}

\author{
Sarah Schmiege, Nancy Felipe Russo
}

\begin{abstract}
Objective To examine the outcomes of an unwanted first pregnancy (abortion $v$ live delivery) and risk of depression and to explain discrepancies with previous research that used the same dataset.

Design Longitudinal cohort study.

Setting Nationally representative sample of US men and women aged 14-24 in 1979.

Participants 1247 women in the US national longitudinal survey of youth who aborted or delivered an unwanted first pregnancy.

Main outcome measures Clinical cut-off and continuous scores on a 1992 measure of the Center for Epidemiological Studies depression scale.

Results Terminating compared with delivering an unwanted first pregnancy was not directly related to risk of clinically significant depression (odds ratio 1.19, 95\% confidence interval 0.85 to 1.66). No evidence was found of a relation between pregnancy outcome and depression in analyses of subgroups known to vary in under-reporting of abortion. In analyses of the characteristics of non-respondents, refusal to provide information on abortion did not explain the lack of detecting a relation between abortion and mental health. The abortion group had a significantly higher mean education and income and lower total family size, all of which were associated with a lower risk of depression.

Conclusions Evidence that choosing to terminate rather than deliver an unwanted first pregnancy puts women at higher risk of depression is inconclusive. Discrepancies between current findings and those of previous research using the same dataset primarily reflect differences in coding of a first pregnancy.
\end{abstract}

\section{Introduction}

Transition to parenthood is an important and stressful life event that involves multiple risk factors (psychological, marital, and economic) for new mothers, ${ }^{1-3}$ particularly when they are young ${ }^{4}$ and the pregnancy is unplanned. ${ }^{6}$ An analysis of the US national longitudinal survey of youth by Reardon and Cougle ${ }^{7}$ reported that women who abort an unintended first pregnancy are at higher risk of clinical depression than those who carry an unintended first pregnancy to term, and that informed consent should warn women of a higher risk of depression if they elect to terminate an unintended pregnancy. The coding and analysis of that trial's data show flaws, however, the most critical of which are misidentification of unwanted first pregnancies and exclusion of women at highest risk of depression associated with early childbearing.

We examined the relation between the outcome of an unwanted first pregnancy (abortion $v$ live delivery) and depression, unadjusted and adjusted for relevant social and personal factors. These analyses test the same hypotheses as that of Reardon and Cougle, ${ }^{7}$ but with more precise coding of variables and appropriate criteria for sample selection.

We then analysed the relation between abortion and depression across groups known to vary in rates of under-reporting of abortion, and we examined the relation between refusals to submit a confidential card containing information on abortion and depression.

Finally, we examined the possibility of indirect relations between pregnancy outcome and depression through the effect of abortion on social outcomes known to relate to mental health-specifically, education, income, and family size (number of children).

As in the previous study, ${ }^{7}$ we selected for pregnancies that were unwanted at the time or not wanted at all. Because these definitions exclude unintended first pregnancies described as wanted or that "didn't matter," we term the pregnancies as unwanted rather than unintended. ${ }^{7}$

\section{Methods}

Participants were women from the US national longitudinal survey of youth (NLSY), a national probability sample of civilian men and women aged 14-21 years in 1979, the year the survey began. The figure shows participant flow from the total survey population of 12686 people to the sample of interest. Of 6283 women, $1820(29 \%)$ were missing data on depression in 1992. Of the 4463 remaining women, 1247 were identified as having an unwanted first pregnancy that ended in a live delivery or abortion. Our sample size is either 1247 or 1004, depending on whether we adjusted for explanatory variables. Year of first pregnancy ranged from 1970 to 1992.

\section{Construction of variables}

The 1992 Center for Epidemiological Studies depression scale consists of 20 questions, each scaled from 0 (rarely) to 3 (most of the time). Scores potentially range from 0 to 60 , with higher scores indicating greater depression. A standard cut-off score dichotomised participants into high (score $>15$ ) or low risk (score $\leq 15$ ) categories. $^{7}$ To more precisely capture the range of depressive symptoms, we carried out parallel analyses using the continuous version of the scale.

All explanatory variables had been used by Reardon and Cougle $^{7}$ and all were significantly correlated with outcome measures in both their and our study. Outcome measures included race, age at first pregnancy, and 1992 measures for education, income, and marital status. Education was a continuous variable based on completion of several grades. Income was an ordered categorical variable ranging from 1-10 (for example, 1 was associated with $\$ 0-10000$ ( $\$ 5700$; €8316) per year, 2 with 
\$10 001-20 000, and 10 with \$90 001 and above). After ensuring no statistically significant differences existed among groups that were never married, divorced, or separated, we dichotomised marital status in 1992 into married or unmarried.

In keeping with the intent of the Reardon and Cougle study, ${ }^{7}$ we identified women whose first pregnancy was unwanted and resulted in a live birth or abortion. To ensure accuracy we constructed our variables using coding language provided by staff of the national longitudinal survey of youth. Respondents indicated the outcome of a first ever pregnancy by interview, beginning in 1983 and continuing across subsequent interviews in 1984 and every two years thereafter. At each interview, participants were asked to identify any pregnancies since their last interview and whether or not the pregnancy was wanted (yes, didn't matter, not then, not ever). We then linked the responses to the outcome of first pregnancy. We excluded from the delivery group women who responded with yes or didn't matter. We assumed that first pregnancies terminated by abortion were unwanted unless otherwise specified; we excluded 15 women in the abortion group who reported that their pregnancies were wanted.

Using this coding approach, we identified a smaller number of eligible women than in the previous study, ${ }^{7}$ even when compared to the corrected numbers reported (http:// bmj.bmjjournals.com/cgi/contentnw/full/324/7345/1097/

Ful). As with the previous study, we identified 4463 women with 1992 scores on the Center for Epidemiological Studies depression scale. Both studies seemed to use the same variable to assess whether the pregnancy was wanted; the difference seems to be in the variables used to identify first pregnancy.

The dataset of the national longitudinal survey of youth is large and complex, with over 100000 variables. A search using the keyword "pregnancy" yields over 3000 variables. Of these, some refer to pregnancy across the lifespan, some refer to pregnancy since the last interview, some are generated by follow-ups to other questions, and some are constructed from combinations of previous answers. Identification of a first unwanted pregnancy is particularly complex as it involves combining answers to a series of "if then" questions across survey years.

Contrary to the previous study, we did not exclude women in the delivery group with subsequent abortions. Our stated purpose was to examine the relation between outcome of a first unwanted pregnancy and later depression, and the exclusion of women with subsequent abortions from either abortion or delivery groups would make generalisation to all women with an unwanted first pregnancy inappropriate. It could further be argued that women who have multiple unwanted pregnancies (whether terminated by delivery or abortion) are more likely to be depressed than other women. Consequently, differential exclusion of women from the delivery group on the basis of subsequent abortion creates a bias in favour of finding lower depression in that group. In any case, conclusions of analyses (not reported here) that excluded all women who aborted subsequent pregnancies did not differ from the results reported below.

\section{Analysis plan}

Analyses were carried out using SAS version 8.2. Before testing the primary study hypotheses, we compared the statistics of the sampling approach used here with that used in the previous study. ${ }^{7}$ Next we examined the relation between pregnancy outcome and depression. Logistic regression analyses predicted cut-off scores on the depression scale $(0=$ non-depressed; $1=$ depressed $)$ from pregnancy outcome $(0=$ delivery; $1=$ abortion), unadjusted and adjusted for explanatory variables. Odds ratios greater than 1 would indicate greater depression in the abortion group, given the coding pattern of variables. We used ordinary least squares regression for parallel analyses predicting continuous scores for depression.

It has been suggested that under-reporting of abortion may lead to failures in detecting a link between abortion and depression. ${ }^{78}$ Because under-reporting has been shown to differ across subgroups of individuals (for example, being married, white, and Protestant have been associated with lower under-reporting), ${ }^{9}$ we examined the relation between pregnancy outcome and depression across the following groups: white versus black married and unmarried women and Catholic versus non-Catholic women. We also addressed the under-reporting of abortion by examining differences in depression between those who did and did not submit the confidential abortion card used by the national longitudinal survey of youth to collect data on abortion. Information on refusals was provided in 1994 and compared with scores on the Center for Epidemiological Studies depression scale. $\chi^{2}$ tests

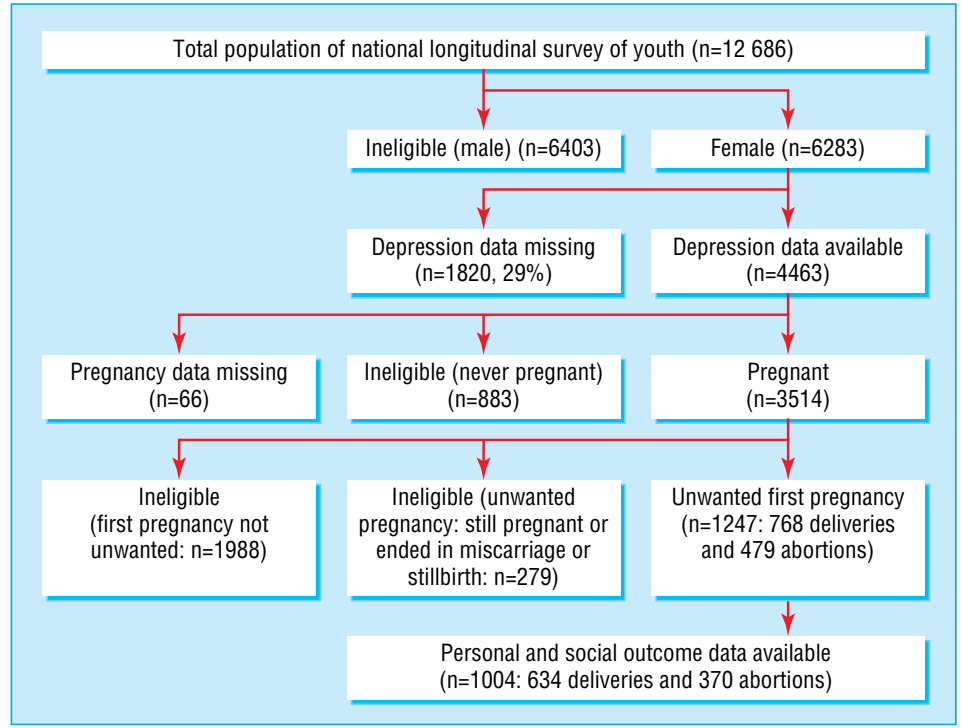

Participant flow from population of national longitudinal survey of youth to sample of interest 
Table 1 Proportion of participants in high risk category for depression and mean continuous scores, stratified by pregnancies before 1980 and those occurring from 1980 onwards

\begin{tabular}{|c|c|c|c|c|c|c|}
\hline Subgroups & $\begin{array}{c}\text { No }(\%) \text { in high risk category for } \\
\text { depression* }\end{array}$ & Odds ratio $(95 \% \mathrm{CI})$ & $P$ value & $\begin{array}{l}\text { Mean (SD) depression } \\
\text { score }\end{array}$ & Mean difference $(95 \% \mathrm{CI})$ & $P$ value \\
\hline \multicolumn{7}{|l|}{ Delivery group: } \\
\hline Before $1980 \dagger$ & 109/310 (35) & & & $13.5(10.6)$ & & \\
\hline After 1980 & $111 / 457$ (24) & 0.59 (0.43 to 0.81$)$ & 0.001 & $10.7(9.4)$ & 2.81 (1.38 to 4.23) & 0.002 \\
\hline \multicolumn{7}{|l|}{ Abortion group: } \\
\hline Before 1980 & $59 / 225(26)$ & 0.66 (0.45 to 0.96$)$ & 0.028 & $10.9(9.6)$ & 2.66 (0.91 to 4.41) & 0.003 \\
\hline After 1980 & $55 / 232(24)$ & 0.57 (0.39 to 0.84$)$ & 0.004 & $10.8(10.4)$ & 2.73 (0.95 to 4.52$)$ & 0.003 \\
\hline
\end{tabular}

$\mathrm{n}=23$ cases missing due to no data on year first pregnancy began.

${ }^{*}$ Center for Epidemiological Studies depression scale score greater than 15 .

†Reference group. For each comparison the pre-1980 delivery group was coded as 0 and each other group coded as 1 so that odds ratios less than 1.0 indicate greater depression in the pre-1980 delivery group.

were used to compare the cut-off scores for depression within the subgroups; continuous scores were compared using $t$ tests. We examined indirect relations between pregnancy outcome and depression by analyses carried out in two parts: $t$ tests were used to compare mean education, family income, and total number of children across delivery and abortion groups, and logistic and ordinary least squares regression were used to examine the three social variables as predictors of depression scores.

\section{Results}

The appropriateness of our sampling approach was assessed over that of the previous study for the testing of the research questions. An abbreviated form of the Rotter internal-external locus of control scale was used in the Reardon and Cougle ${ }^{7}$ study in an attempt to account for "prior psychiatric state." Because this scale was administered in 1979, the authors excluded all women who had experienced their first pregnancy before that time, including most (339 of 425) teenage pregnancies.

We examined the relation between depression and pregnancy outcome, stratified by a dichotomous variable for year of pregnancy (before $1980 v 1980$ and beyond), to statistically compare the two sampling approaches (table 1). We examined the differences in risk of depression among the four groups by using the pre-1980 delivery group as a reference point for comparison with the other three groups. Women in the pre-1980 delivery group had a significantly greater risk of experiencing depression than women in the other three groups.

Excluding women with first pregnancies before 1980 consequently removed women at highest risk for negative outcomes associated with early childbearing, making it inappropriate to generalise findings from the previous research to all first pregnancies. Primary analyses for our study were thus based on all women for whom unwanted first pregnancies could be identified. For comparison purposes with Reardon and Cougle's study, we also carried out analyses predicting depression scores from pregnancy outcome on a subsample of women $(n=689)$ whose pregnancy occurred between 1980 and 1992, adjusting for 1979 Rotter scores.

\section{Prediction of depression from pregnancy outcome}

Pregnancy outcome did not predict depression scores in either the full sample or in the subsample of pregnancies occurring between 1980 and 1992, even when adjusted for personal and social indicators (table 2). Examination of the raw data confirms similar scores for depression across the delivery and abortion groups, with $28.6 \%$ of participants in the delivery group compared with $24.8 \%$ in the abortion group being in the high risk category (mean depression scores 11.8 and 10.8, respectively).

\section{Under-reporting of abortion}

We found no evidence for an association between abortion and depression across the subgroups (table 3).

Table 2 Odds ratios (95\% confidence intervals) predicting depression cut-off scores from pregnancy outcome and $\beta$ coefficients (SE) showing mean differences in depression continuous scores by pregnancy outcome, in full sample and subsample of post-1979 pregnancies, unadjusted and adjusted for explanatory variables

\begin{tabular}{|c|c|c|c|c|c|c|c|c|c|c|}
\hline \multirow[b]{2}{*}{ Sample and group } & \multicolumn{5}{|c|}{ Logistic regression predicting depression cut-off scores* } & \multicolumn{5}{|c|}{$\begin{array}{l}\text { Ordinary least squares regression predicting depression continuous } \\
\text { scores }\end{array}$} \\
\hline & $\begin{array}{c}\text { No }(\%) \\
\text { exceeding } \\
\text { cut-off score }\end{array}$ & $\begin{array}{l}\text { Unadjusted odds } \\
\text { ratio }(95 \% \mathrm{CI})\end{array}$ & $P$ value & Adjusted & $P$ value & Mean (SD) & $\begin{array}{l}\text { Unadjusted } \beta \\
\quad(\mathrm{SE})\end{array}$ & $P$ value & Adjusted $\beta$ (SE) & $P$ value \\
\hline \multicolumn{11}{|l|}{ Full sample: } \\
\hline $\begin{array}{l}\text { Delivery group } \\
(\mathrm{n}=768)\end{array}$ & $220(28.6)$ & 0.82 (0.64 to 1.07$)$ & 0.14 & $1.19(0.85$ to 1.66$) \dagger$ & 0.30 & $11.8(9.95)$ & $-1.07(0.58)$ & 0.06 & $0.38(0.68)^{\star}$ & 0.58 \\
\hline $\begin{array}{l}\text { Abortion group } \\
(\mathrm{n}=479)\end{array}$ & $119(24.8)$ & - & & - & & $10.8(9.9)$ & - & & - & \\
\hline \multicolumn{11}{|l|}{$\begin{array}{l}\text { Post-1979 } \\
\text { pregnancies: }\end{array}$} \\
\hline $\begin{array}{l}\text { Delivery group } \\
(\mathrm{n}=457)\end{array}$ & $111(24.3)$ & 0.97 (0.67 to 1.40$)$ & 0.87 & $1.33(0.84$ to 2.10$) \ddagger$ & 0.23 & $10.7(9.4)$ & $0.07(0.78)$ & 0.93 & $0.92(0.87) \dagger$ & 0.29 \\
\hline $\begin{array}{l}\text { Abortion group } \\
(\mathrm{n}=232)\end{array}$ & $55(23.7)$ & - & & - & & $10.8(10.4)$ & - & & - & \\
\hline
\end{tabular}

Pregnancy outcome coded 0 for delivery and 1 for abortion; higher depression scores indicate greater levels of depression. Odds ratios greater than 1.0 and positive $\beta$ coefficients indicate higher risk of depression in abortion group.

*Greater than 15.

†Adjusted for race, age at first pregnancy, and 1992 marital status, education, and family income.

$\Varangle$ Adjusted for 1979 measure of Rotter internal-external locus of control, race, age at first pregnancy, and 1992 marital status, education, and family income. 
Table 3 Relation between depression and pregnancy outcome within subpopulations known to vary in amount of under-reporting of abortion

\begin{tabular}{lcccccccc} 
& & & & & & & \multicolumn{2}{c}{ Mean (SD) continuous depression } \\
score*
\end{tabular}

*Depression measured on Center for Epidemiological Studies depression scale. Pregnancy outcome coded 0 for delivery and 1 for abortion; higher scores indicate greater levels of depression. Cut-off score greater than 15.

Among the 4306 women in the larger sample who had both a depression score and abortion card information, $24.5 \%$ of women $(1025 / 4190)$ who submitted the card were in the high risk group compared with $12.9 \%(15 / 116)$ of women in the refusal group $\left(\chi^{2}=8.19, \mathrm{P}=0.004\right)$. The mean continuous score for the refusal group was significantly lower than that for the group that submitted the card $(7.9 v 10.7 ; t=2.99, \mathrm{P}=0.003)$.

\section{Indirect effects analyses}

We found no evidence that terminating compared with delivering an unwanted first pregnancy changes risk for subsequent depression. This does not mean that the outcome of an unwanted first pregnancy has no relation to risk of depression. The indirect effects of pregnancy outcome on depression were examined, through effects of abortion on income, education, and number of children.

The abortion group had significantly higher mean education and income and lower total family size (table 4), findings that are consistent with previous analyses of the national longitudinal survey of youth. ${ }^{10}$

Separate regression analyses then examined each social variable (with pregnancy outcome also included in each model) as predictors of depression cut-off and continuous scores (table 4). Higher education and income predicted a lower risk of depression and larger family size predicted a higher risk of depression.

\section{Discussion}

Our results provide no support for the claim by Reardon and Cougle $^{7}$ that terminating an unwanted first pregnancy contributes to risk of subsequent depression. Instead, our finding that the group that delivered before 1980 had a significantly higher risk of depression than all other groups directly contradicts the claim that terminating an unwanted first pregnancy puts women at higher risk of subsequent depression, particularly for younger women. These results cannot be reason- ably explained by underreporting of abortion. Indeed, finding that depression scores for the group that refused to fill out a confidential abortion card were significantly lower than for the reporting group suggests that women who are willing to disclose abortion are also more willing to disclose stigmatising mental health problems, such as depressive symptoms-that is, they exhibit a form of "over-reporting bias."

The observed association of abortion with education and income-social variables that have profound implications for mental health-is consistent with the literature on the negative effects of early and unwanted childbearing. ${ }^{1-6}$ Providing pregnant women with informed consent about the risks and benefits of alternatives for resolving unwanted pregnancy requires an understanding of the potential for both direct and indirect effects of the full range of alternatives, as well as the ability to apply that knowledge to the patient's unique characteristics and circumstances.

Some women who undergo abortion will also experience clinical levels of depression. However, other research has found that pre-existing mental health is the more important predictor of mental health after pregnancy, regardless of how the pregnancy is resolved..$^{11}$ If the goal is to understand the factors that affect the mental health of women, consideration of the range of influences that contribute to risk and resilience is more likely to be successful than a single minded focus on risks of abortion.

Inconsistencies between our findings and those from the Reardon and Cougle ${ }^{7}$ study can primarily be explained by differential coding of key variables and sample selection. Given that the numbers used in our study are based on coding language used by staff of the survey, we believe they provide the most accurate variable definition. The previous study's exclusion of a major proportion of adolescent pregnancies is a fatal flaw for any study attempting to generalise findings to first unwanted pregnancies.

Table 4 Indirect effect analyses to examine differences in mean (SD) education, income, and family size across delivery and abortion groups and to examine education, income, and family size as predictors of cut-off scores for depression (logistic regression) and continuous scores for depression (ordinary least squares regression)

\begin{tabular}{lccccccccc} 
Variable & Delivery group & Abortion group & $\begin{array}{c}\boldsymbol{t} \text { test comparing } \\
\text { groups }\end{array}$ & P value & Odds ratio (95\% CI) & P value & $\boldsymbol{\beta}$ (SE) & P value \\
\hline Education & $12.25(2.07)$ & $13.34(2.33)$ & 8.70 & $<0.001$ & $0.83(0.78$ to 0.89$)$ & $<0.001$ & $-0.764(0.128)$ & $<0.001$ \\
\hline Income & $3.15(2.10)$ & $4.15(2.44)$ & 7.06 & $<0.001$ & $0.77(0.72$ to 0.84$)$ & $<0.001$ & $-1.034(0.131)$ & $<0.001$ \\
\hline Family size & $2.68(1.21)$ & $1.24(1.17)$ & -20.80 & $<0.001$ & $1.13(1.02$ to 1.25$)$ & $<0.05$ & $0.752(0.234)$ & $<0.001$ \\
\hline
\end{tabular}

Pregnancy outcome coded 0 for delivery and 1 for abortion.

For income variable, mean of 3.15 corresponds to income in range $\$ 20001$ ( $£ 11400 ; € 16632$ ) to $\$ 30000$, and mean of 4.15 corresponds to income range $\$ 30001$ to $\$ 40000$. Positive $t$ values comparing variables across groups indicate higher scores in abortion group; negative values indicate lower scores in abortion group.

Odds ratios less than 1 and negative $\beta$ coefficients indicate that greater income and education are associated with decreased depression; larger family size is associated with increased depression, as evidenced by the odds ratio greater than 1 and positive $\beta$ coefficient. 
Several limitations remain, however. The research focuses on first pregnancies and does not encompass unwanted pregnancies experienced by women who previously had either a planned pregnancy or an unintended pregnancy that was wanted or that didn't matter. The process selected only women whose unwanted first pregnancies ended in abortion or a live birth, excluding women who had a stillbirth, miscarriage, or who were pregnant at the time of their most recent interview. In addition, recall difficulties of participants were likely more of an issue here than in the previous study ${ }^{7}$ because the total sample included pregnancies occurring before 1980. Questions assessing the want and outcome of first pregnancy were first included in the national longitudinal survey of youth battery in 1983 and 1984, respectively. The time between pregnancy and interview was, at most, four years for pregnancies occurring after 1980, but up to a decade for pregnancies before 1980. It is not clear whether time lag might present as an overestimation or underestimation of the number of unwanted pregnancies. Furthermore, the dataset had missing data. Only 272 of the 1820 women missing data on depression were, however, identified as eligible (having an unwanted first pregnancy). Finally, for some participants the time from first pregnancy to measurement of depression was long, with the time varying across participants, complicating the interpretation of a causal relation. In the intervening time, many factors that both reflect and contribute to risk of depression can occur, including changes in educational and income levels, employment and marital status, and number of pregnancies and their outcomes. This limitation would be of more concern had there been an association between pregnancy outcome and depression in need of explanation, however.

We conclude that, under present conditions of legal access to abortion, there is no credible evidence that choosing to terminate an unwanted first pregnancy puts women at higher risk of subsequent depression than does choosing to deliver an unwanted first pregnancy. Delivering a first unwanted pregnancy is, however, associated with lower education and income and larger family size-all risk factors for depression. This suggests that if the goal is to reduce women's risk for depression, research should focus on how to prevent and ameliorate the effect of unwanted childbearing, particularly for younger women.

We thank Canada Keck (national longitudinal survey of youth) for providing the coding language from which we constructed the variables for first pregnancy outcome and wantedness and Jean Denious for her comments on earlier drafts of the paper.

Contributors: SS and NFR jointly contributed to the design, analysis, interpretation, and writing of the study. SS and NFR are guarantors.

Funding: The national longitudinal survey of youth survey is carried out by the Center for Human Resource Research, Ohio State University, and is funded by the Bureau of Labor Statistics, United States Department of Labor.

Competing interests: None declared.

Ethical approval: This study was approved by the institutional review board of Ohio State University.

\section{What is already known on this topic}

Well designed studies have not found that abortion contributes to an increased risk of depression

A recent study based on data from the US national longitudinal survey of youth reports a relation between termination of unwanted first pregnancy and risk of depression

\section{What this study adds}

The previous relation between termination of unwanted first pregnancy and risk of depression was not found when more appropriate coding and sampling approaches were applied to the same dataset

Abortion may be indirectly associated with a lower risk of depression through beneficial effects on education, income, and control of family size

Women who are willing to disclose abortion may also be more willing to disclose depressive symptoms, exhibiting a form of "over-reporting bias"

Under-reporting of abortion may occur, but does not seem to account for lack of detection of a relation between abortion and depression

1 Campbell SB, Cohn JF, Meyers TA. Course and correlates of post-partum depression during the transition to parenthood. Dev Psychopath 1992;7:29-47.

2 Cowan SP, Cowan PA, Heming G, Garrett E, Coysh WS, Curtis-Boles H, et al. Transition to parenthood: is, hers, and there's. J Fam Issues 2000;6:451-81.

3 Glenn ND, McLanahan S. Children and marital happiness: a further specification of the relationship.J Marriage Fam 1982;44:63-72.

4 Horwitz SM, Klerman L, Kuo HS, Jekel J. School age mothers: predictors of long-term educational and economic outcomes. Pediatrics 1991;87:862-8.

Trad PV. Assessing the patterns that prevent teenage pregnancy. Adolescence 1999;34:221-40.

6 Cox MJ, Paley B, Burchnal M, Payne CC. Marital perceptions and interactions across the transition to parenthood.J Marriage Fam 1999;61:611-25.

7 Reardon DC, Cougle JR. Depression and unintended pregnancy in the National LonReardon DC, Cougle JR. Depression and unintended pregnanc
gitudinal Survey of Youth: a cohort study. BMJ 2002;324:151-2.

Reardon DC. Clinical depression after unintended pregnancy link to abortion. http:// www.afterabortion.org/news/depressionbmj.html

Jones EF, Forrest JD. Underreporting of abortion in surveys of U.S. women: 1976 to 1988. Demography 1992;29:113-26.

10 Russo NF, Zierk K. Abortion, childbearing, and women's well-being, Prof Psychol Res Pr 1992;23:269-80.

11 Gilchrist AC, Hanaford PC, Frank P, Kay CR. Termination of pregnancy and psychiatric morbidity. Brit J Psych 1995;167:243-8.

(Accepted 16 September 2005)

doi $10.1136 /$ bmj.38623.532384.55

Department of Psychology UCB 345, University of Colorado, Boulder, CO 80309-0345, USA

Sarah Schmiege postdoctoral research associate

Department of Psychology, Arizona State University, Box 871104, Tempe, AZ 85287-1104, USA

Nancy Felipe Russo regents professor

Correspondence to: N F Russo nancy.russo@asu.edu 